\title{
COMUNICACIÓN
}

\section{Trichinellosis en perros callejeros de la ciudad de Zacatecas México}

\author{
VICENTE BERUMEN DE LA TORRE, JOSÉ J. \\ MUÑOZ ESCOBEDO, y MARÍA A. MORENO GARCÍA
}

TRICHINELLOSIS IN DOGS FROM IN ZACATECAS, MÉXICO

In this study 51 tongues from dogs used for practices at the Universidad Autonoma the Zacatecas were inspected for the presence of Trichinella spiralis, through the muscle compression and artificial digestionmethods. The presence of the parasite was found in 3 tongue indicating that trichinellosis is an important public health ploblem in Zacatecas and therefore special attention to pig inspection and proper disposal of infected meat should then be given at slaughterhouses in order to prevent the transmission of this important zoonosis.

Key words: Trichinellosis, Trichinella spiralis, Dogs parasites, Mexico.

\section{INTRODUCCIÓN}

Trichinella spiralis es un nematodo zoonótico capaz de invadir e infectar los músculos de diferente mamíferos, entre ellos el perro, el cuál cuando come carne con sus larvas puede infectarse y presentar un cuadro clínico manifestado por anorexia emaciación y dolor muscular, En infecciones superiores a las 100.000 larvas infectantes $\left(\mathrm{L}_{1}\right)$ puede producirse la muerte del animal ${ }^{1}$. La trichinellosis se puede clasificar como una amfixenosis ya que afecta por igual a humanos como animales, pero también se le puede clasificar como una zoonosis directa, ya que requiere de un huésped vertebrado para mantener el agente infeccioso ${ }^{3}$.

La presencia de $T$. spiralis en perros podría significar que se infectaron con la ingestión de productos cárnicos de consumo humano, y por consiguiente tener una fuente común de infección ${ }^{2}$.

Si bien es cierto que el perro no solo come desechos alimenticios, sino que puede alimentarse de carne de otros animales (como por ejemplo de roedores), o de vísceras crudas que se botan alrededor de los mataderos sean o no clandestinos, algunas de sus infecciones producidas con $T$. spiralis deben haberse originado por consumo de restos de alimentos del hombre.

En los Estados de Zacatecas, Durango, Chihuahua, Guanajuato, Jalisco, Estado de México y el Distrito Federal se han registrado brotes epidémicos de trichinellosis ${ }^{4}$. En el esta-

Centro de Biología Experimental. Universidad Autónoma de Zacatecas.

Apartado Postal 12.

98600 Guadalupe, Zac. México. 
do de Zacatecas el primer brote se registró en 1978 en donde se infectaron 18 personas y 5 de ellas murieron. ${ }^{5}$

El presente trabajo tiene por objeto medir la magnitud de las infecciones en perros. En la ciudad de Zacatecas.

\section{MATERIAL Y MÉTODOS}

Se obtuvieron 51 lenguas y 29 sueros, de perros de vida libre que fueron sacrificados en el Departamento de Farmacología de la Unidad Académica de Medicina Humana y Ciencias de la Salud de la Universidad Autónoma de Zacatecas. Una vez terminada la práctica de farmacología, se procedió a obtener sangre de la vena cefálica o de la arteria safena, y luego de sacrificarlos humanitariamente con pentobarbital sódico inyectado directamente en el corazón, se les extrajo sus lengua para estudios de trichinellosis. Dentro de 24 horas se realizaron las técnicas de diagnóstico para $T$. spiralis de compresión y de digestión artificial. A la sangre se le separó el suero utilizando por centrifugación a 3.000 r.p.m. durante 8 minutos y el suero obtenido fue almacenado y congelado en alícuotas hasta su uso. Posteriormente a los sueros se les realizó la técnica inmunológica de Microinmunodifusión doble (MIDD) ${ }^{4}$, Como antígeno se utilizó el antígeno soluble total (A.S.T.) de triquina obtenido por nitrógeno líquido.

A 5 g. de la musculatura molida de la base de la lengua de cada animal se le realizó una digestión artificial por $38^{\mathrm{a}}$ Cpor 24 horas en una solución de $7 \mathrm{~g}$ de pepsina de 10.000 unidades en 2 litros de agua tibia más $20 \mathrm{ml}$ de ácido clorhídrico siguiendo procedimientos estandarizados $^{6}$. En el sedimento de estas digestiones se observó al microscopio óptico de luz a 10X, 20X, 40X o seco débil la presencia de larvas de $T$. spiralis.

También se procedió a revisar bajo microscopio óptico de luz, la presencia de larvas en las lenguas de los animales mediante la compresión de pequeños trozos de carne $(10 \mathrm{mg})$ entre dos portaobjetos sujetados con cinta adhesiva. Este procedimiento se llevó a cabo por triplicado y de 3 sitios diferentes de la lengua. $^{7}$
Con las lenguas infectadas con $\mathrm{L}_{1}$ de $T$. spiralis se infectaron 3 ratas (Rattus norvergicus), cepa Long Evans, hembras de 2 meses de edad, tratando de administrar por vía oral, aproximadamente una $100 \mathrm{~L}_{1}$.

\section{RESULTADOS}

De las 51 lenguas a las que se les realizó las técnicas directas de compresión y digestión artificial, $3(5,82 \%)$ fueron positivas a infección por T. spiralis (Figura 1). Las MIDD efectuadas resultaron negativas. Finalmente cabe mencionar que se reprodujo el ciclo vital de $T$. spiralis en el modelo murino.

\section{DISCUSIÓN}

De las 51 lenguas de perros colectadas en el Departamento de Farmacología de la Unidad Académica Medicina Humana y Ciencias de la Salud, de la U.A.Z, $3(5,82 \%)$ salieron positivas con infección con $T$. spiralis lo que permite definir que el parásito se encuentra en perros doméstico, con mayor frecuencia de lo que podría pensarse.

Al encontrarse el parásito en perros nos permite suponer, que se infectaron a partir de consumir desperdicios cárnicos crudos o mal cocidos entregados por sus dueños o bien que han consumido restos de animales en la vía pública o en basurales. De ser la primera de estas vías de infección vale la pena recomendar la intensificación de las medidas de control

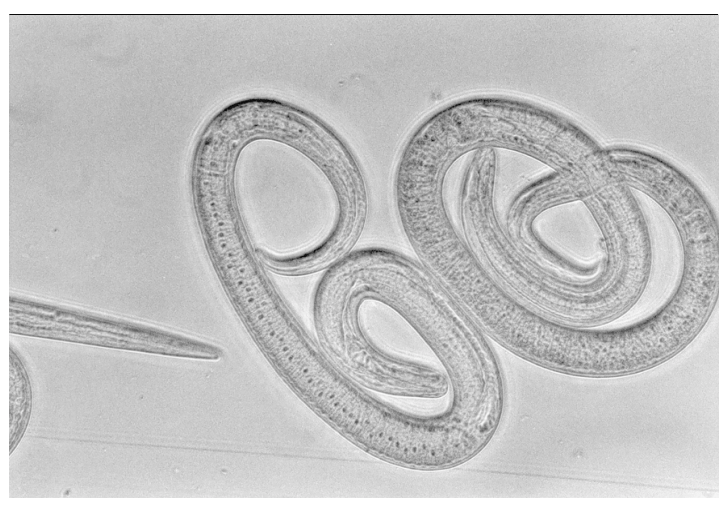

Figura 1. L I de Trichinella spiralis obtenidas de lengua de perro por la Técnica Directa de Digestión Artificial, observadas al microscopio óptico de luz a 40X. 
sanitario en los mataderos y en la venta de la carne de cerdo de abasto.

La técnica de Digestión Artificial tiene una efectividad alrededor del $90 \%$ esta técnica tiene como ventaja ser barata, su desventaja es que es tardada para utilizarse en el rastro. La técnica de Compresión tiene una efectividad de alrededor del $50 \%$, su ventaja es que es barata y rápida y su desventaja es su baja efectividad. ${ }^{8}$

Ya se sabe ampliamente en el campo de la inmunología y aquí nuevamente lo evidenciamos, la MIDD resultó negativa, debido a que es una técnica indirecta específica pero de baja sensibilidad detecta miligramos de proteína ${ }^{9}$. Consideramos, que se debe buscar técnicas serológicas adecuadas y rápidas para implementarla en los mataderos misma de tal manera que se entreguen para el consumo carnes seguras y que no altere la rutina de la industria de la carne. Nosotros proponemos la Dot-Elisa que es sensible y se puede llevar a cabo en poco tiempo y su costo no es alto, nuestra experiencia al respecto ha sido satisfactoria ${ }^{9}$.

\section{RESUMEN}

Se inspeccionaron 51 lenguas de perros procedentes de la ciudad de Zacatecas, México y se detectaron $3(5,82 \%)$ positivas a infección por Trichinella spiralis mediante las técnicas de compresión y digestión artificial. Al encontrar el parásito en el perro significa que éste muy posiblemente consumió desperdicios de comida de humanos, aunque el perro también pudo haber comido cadáveres de animales.

\section{REFERENCIAS}

1.- ACHA N, SZYFRES B. Zoonosis y enfermedades transmisibles comunes al hombre y a los animales. $2^{\text {a }}$ Edición. Organización Panamericana de la Salud. Editado en Washigton D.C. USA. 1997; 865-9.

2.- MIKHAIL E M, MANSOUR N S, AWDALLA H N. Identification of Trichinella isolates from natural infected stray dogs in Egypt. J Parasitol 1994; 80: 151-4.

3.- GARCÍA VÁZQUEZ Z. Epidemiología Veterinaria y Salud Animal. $1^{\text {a }}$ Edición. Editorial Limusa. México. 1990; 87-98.

4.- FLORES CÁRDENAS G, MORENO GARCÍA M A. Detección de Trichinella spiralis por DOT-ELISA en sueros de cerdos. Revista Latinoamericana de Microbiología 2000; supplement II 42: 100-1.

5.- FRAGOSO R, TAVIZÓN $P$, VILLACAÑA F $H$. Un brote de Triquinelosis (triquinelosis) en Laguna de Carretero, Zacatecas. Salud Pública. México. 1981; XXIII: 25-41.

6.- LABORATORIO GENERAL CENTRAL. Manual de Técnicas de Parasitología Veterinaria. Weybridge, Gran Bretaña. Edit. Her Majesty's Station Office. Zaragoza España. Traducido al Español por Editorial Acribia 1971; 83-4.

7.- BERUMEN DE LA TORRE V. Manual de Prácticas de Parasitología Veterinaria. Laboratorio de Parasitología Veterinaria de la Unidad Académica Medicina Veterinaria. U. A Z. 1998.

8.- RIBICICH M, ROSA A, MOLINA V et al. Comparación entre la Digestión Artificial y la Triquinoscopía para la detección de Trichinella spiralis en carne porcina. Investigación Veterinaria. Buenos Aires Argentina. 2000; 2: 81-8.

9.- MORENO G, VACIO DE LA T, REVELES H, MUÑOZ E. Epidemiologíade T. spiralis en el estado de Zacatecas, México. J Brasil Patol 2001; 37: 25.

Agradecimientos: A la Dra en C. Patricia Yahuaca Mendoza, Jefe del Departamento de Farmacología de la Unidad Académica de Medicina Humana y Ciencias de la Salud de la Universidad Autónoma de Zacatecas. 